\title{
Loss-of-function mutations in TBK1 can cause familial ALS
}

New research published in Nature

Neuroscience implicates specific variants of the TANK-binding kinase 1 (TBK1) gene in familial amyotrophic lateral sclerosis (ALS). The exome-sequencing study could have wide implications for the study of ALS, and might facilitate new approaches to diagnosis and treatment.

\section{Our findings ... link three ALS genes to one common pathway for autophagy regulation... 77}

“Currently known ALS-related genes explain only a minority of cases, but have told us a lot about the basic principles of ALS pathogenesis, such as disturbed RNA metabolism or pathological protein aggregation," explains Jochen Weishaupt, co-supervisor of the new study.

The investigators recruited 252 patients with familial ALS who had previously tested negative for SOD1 and C9orf72 mutations. The patients then underwent whole-exome sequencing, and these data were compared with sequences from 827 controls who had no family history of ALS.

Eight loss-of-function variants of TBK1 were identified as unique to the group of patients with ALS, and achieved genomewide significance for their association with this condition. A subsequent targeted screen for TBK1 mutations in healthy controls and patients with sporadic ALS suggested that TBK1 loss-of-function mutations were mostly specific to familial ALS.

The investigators then performed a series of in vitro tests to determine how these mutations might lead to disease. In cell lines derived from the patients with familial ALS, most of the TBK1 loss-offunction mutations led to substantial reductions in the expression of TBK1 mRNA and protein. Another ALS-linked mutation only truncated the C-terminus of the protein.

"On the basis of our genetic and functional findings, we could delineate mutations that affected the C-terminal coiled-coil domain 2 of TBK1 as sufficient to cause neurodegeneration," explains Weishaupt. He also points out that TBK1 interacts closely with optineurin and sequestosome-1 (also known as ubiquitinbinding protein $\mathrm{p} 62$ ), which have both been implicated in ALS pathogenesis.

"Our findings thus link three ALS genes to one common pathway for autophagy regulation," concludes Weishaupt. This pathway might represent a therapeutic target for patients with ALS.

In the paper, the investigators also present the first autopsy data from a patient with familial TBK1-ALS, who had perinuclear inclusions of TAR DNAbinding protein 43 and sequestosome-1 in areas of the temporal lobe. Further postmortem analyses will be necessary to confirm the pathology of this form of familial ALS.

Alex Chase

Original article Freischmidt, A. et al. Haploinsufficiency of TBK1 causes familial amyotrophic lateral sclerosis and fronto-temporal dementia. Nat. Neurosci. doi:10.1038/ nn. 4000 